\title{
Effect of Some Fabric and Sewing Conditions on Apparel Seam Characteristics
}

\author{
A. K. Choudhary ${ }^{1}$ and Amit Goel ${ }^{1,2}$ \\ ${ }^{1}$ Department of Textile Technology, National Institute of Technology, Jalandhar, Punjab 144011, India \\ ${ }^{2}$ Punjab Institute of Textile Technology, Amritsar, Punjab 144011, India \\ Correspondence should be addressed to A. K. Choudhary; choudharyak@nitj.ac.in
}

Received 19 April 2013; Accepted 29 May 2013

Academic Editor: Phillip W. Gibson

Copyright (c) 2013 A. K. Choudhary and A. Goel. This is an open access article distributed under the Creative Commons Attribution License, which permits unrestricted use, distribution, and reproduction in any medium, provided the original work is properly cited.

\begin{abstract}
Many previous studies showed that seam appearance as well as performance depends on the interrelationship of fabrics, threads, and the stitch/seam selection and lastly on the sewing conditions, which include the needle size, needle thread tension, stitch density, and the appropriate operation and maintenance of the sewing machine. Present investigation elucidates the effect of blend composition, sewing thread size, and sewing needle parameters on garment seam characteristics (i.e., seam strength, seam strength efficiency, seam puckering, seam stiffness, and drape coefficient). The seam quality characterization is studied through the L9 orthogonal design methodology. In good quality apparels, compatibility of the seams with the functional requirement is very important for serviceability and life of the apparel. Reversibility as well as repairing of seams in the apparel is very limited in the condition of seam failure. Seam strength efficiency is higher for the uniform fiber matrix in the structure of fabrics. The polyester dominated suiting fabrics give minimum seam stiffness because polyester component has low flexural rigidity. The cotton dominated suiting fabrics have less seam puckering due to increase in fabric stiffness.
\end{abstract}

\section{Introduction}

The fabric quality influences not only the quality of the garment but also the ease with which a shell structure can be produced out with flat fabric. The specifications of fabrics for apparel manufacturing can be considered in terms of primary and secondary quality characteristics. The primary quality characteristics are static physical dimensions and secondary characteristics are the reactions of the fabric to an applied dynamic force. The apparel manufacturer is primarily interested in the secondary characteristics of the fabric and focus on the seam quality during the fabrication and production of apparel $[1,2]$. On the other hand, apparel consumers are mainly paying attention to appearance, comfort, and wearability of fabric and evaluate seamquality based on the seam appearance and its mechanical intactness after wear and care procedures of apparel. The production of garments from high quality fabrics not only gives comfort to the wearer but also helps in the smooth working of manufacturing processes and leads to almost defect-free garments. Further, the primary function of a seam is to provide uniform stress transfer from one piece of fabric to another, thus preserving the overall integrity of the fabric assembly. For proper appearance, seam should not contain any defects including skipped stitches, unbalanced stitches, seam grin, distortion or unevenness or puckering, unsteadiness, improper drapeability, uneven seam density, and yarn severance or damage. The overall quality of a seam depends on its strength, elasticity, durability, stability, and appearance [3]. The seam characteristics parameters are seam strength, seam pucker, seam stiffness, seam appearance, and seam efficiency $[4,5]$. According to many researchers $[6,7]$, there are various factors affecting the seam quality. These comprise sewing thread, sewing condition, and others.

A sewing thread is a strong, smooth, evenly spun, hard twisted and elastic, ply or cabled yarn treated by special finishing process to make it resistant to abrasion in its passage through the eye of the needle and through materials in seaming and stitching operation. Sewing thread may be 
TABLE 1: Characteristics of medium weight suiting fabrics.

\begin{tabular}{|c|c|c|c|c|c|c|c|c|c|}
\hline \multirow{3}{*}{$\begin{array}{l}\text { Samples } \\
\text { number }\end{array}$} & \multirow{3}{*}{$\begin{array}{l}\text { Fabric blend } \\
\text { composition (\%) }\end{array}$} & \multirow{3}{*}{$\begin{array}{l}\text { Fabric thickness } \\
\qquad(\mathrm{mm})\end{array}$} & \multirow{3}{*}{$\begin{array}{l}\text { Fabric weight } \\
\text { (GSM) }\end{array}$} & \multicolumn{6}{|c|}{ Thread characteristics } \\
\hline & & & & \multicolumn{2}{|c|}{ Fractional cover } & \multicolumn{2}{|c|}{ Thread density (per inch) } & \multicolumn{2}{|c|}{ Yarn linear density $(\mathrm{Ne})$} \\
\hline & & & & Warp & Weft & Warp & Weft & Warp & Weft \\
\hline 1 & Poly/Cot: 65/35 & 0.44 & 205 & 22.36 & 13.41 & 100 & 60 & 20 & 20 \\
\hline 2 & Poly/Cot: 15/85 & 0.42 & 210 & 22.36 & 13.5 & 100 & 54 & 20 & 16 \\
\hline 3 & Cotton: 100 & 0.40 & 215 & 22.36 & 13.5 & 100 & 54 & 20 & 16 \\
\hline
\end{tabular}

constructed from staple yarn, continuous filament, or core spun yarns. The sewing thread plays a significant role for improving even though it usually represents much less than $1 \%$ by mass of a garment. The high strength of sewing thread always gives better functional performance of seam, namely, seam strength and seam strength efficiency. The different manufacturing parameters of sewing thread are type, ply, finish, twist, and size. The spun-polyester, 3-ply, normal twist, and standard finish sewing thread quality is widely used for all types of sewn fabrics [8]. However, the size of the sewing thread is the most crucial for the stability of seam in apparel, as the improper use of sewing thread size directly affects the seam quality of apparels. Rengasamy et al. [9] established that sewing thread type has a great influence on seam efficiency. He explained that seam efficiency of sewn fabric stitched with polyester textured thread lies in between those of fabric stitched with cotton and spun polyester threads, but more closer to fabric stitched with spun polyester threads. A few researchers conducted research on the effect of thread finishes on seam quality. In the course of garment manufacturing, for protective measures to prevent the thread strength reduction and/or breakage during sewing from the needle heat up, the different types of finishes can be applied over sewing thread according to the specific end use of apparel products. Similarly, Sundaresan et al. [10] found that sewing thread size and ply are the most influencing factors for the seam strength and seam puckering. Higher sewing thread size (Tex value) was subjected to greater friction during sewing operation, which ultimately reduces its strength leading to poor seamstrength and also greater seam puckering. On the contrary, more number of ply in a sewing thread helps to increase the strength of a sewing thread and leads to higher seam strength and/or seam efficiency with a less chance of seam slippage. But the diameter becomes high with more number of ply causing the structural jamming along the seam line, which in turn leads to seam puckering and change in draping behavior of apparel product due to increased stiffness. There is a trend in the apparel industry to select the lowest size of thread with the greatest possible strength for making a seam which is less visible and has good seam functional performance.

The fabric properties which affect the seam quality of apparel are cover factor, weight, thickness, strength, shrinkage, functional finishes, extensibility, bending rigidity, and shear rigidity, some of which form an integral part of low stress mechanical properties. The role of fibre types and its \% in made-up fabric is very important for sewing performances and characteristics due to its effect on made-up fabric mechanical properties. In present experimental work, we have taken polyester-cotton blend and $100 \%$ cotton to see the influences of polyester and cotton fibre on stitching qualities and performances. The L9 orthogonal experimental design is taken into account to see the effect of blend composition, needle thread tension, needle size, and sewing thread linear density on the stitching performances like seam efficiency, seam stiffness, seam puckering, and drape coefficient of seam, as incorporating larger influencing factors and output effects through statistical analysis.

\section{Materials and Methods}

2.1. Materials. Commercially available three types of suiting fabrics of medium weight construction, varying in the blend composition of polyester and cotton components, are taken into consideration for this work. The details of suiting fabric are $210 \mathrm{~g} / \mathrm{m}^{2}, 2 / 1$ Twill, 100 ends/inch (EPI), and 60 picks/inch (PPI). The other characteristics of the fabrics are shown in Table 1.

The GSMs of fabrics are calculated with GSM cutter and electronic weighing balance. The ASTM-D105911 methodology is used for fabric yarn count determinations. The fabric thickness is measured under constant compressive load of 2 KPa with the thickness tester (Karl Schröder KG). The fabric yarn densities are measured according to ASTM-D3375 [11]. Commercially available polyester/cotton core spun sewing threads of different linear densities (40,60, and 80 Tex) are used. These sewing threads are used in the experimental work due to their least reversible strain as compared to cotton sewing threads. The threads are characterized for actual linear density, number of plies, twist direction, and tensile properties. The twist per unit length is measured by direct counting method according to ASTM-D1423 [11], and the tensile testing of the threads is performed at a gauge length of $250 \mathrm{~mm}$ on Zwick tensile testing machine at $35 \mathrm{~mm} /$ minute (corresponding to $20 \mathrm{~s}$ breaking time), as per ASTM-D2256 [11]. The number of test of 30 is carried out and the error is less than $4 \%$ at $95 \%$ confidence level. The specifications of the sewing threads are shown in Table 2.

The most appropriate needle sizes are used in stitching works (i.e., Organ 11, 14, and 16) and the stitch density is kept constant at 10 stitches per inch (SPI). The details of the needle dimensions are given in Table 3.

2.2. Seam Preparation. All stitching work has been done on Juki industrial lockstitch sewing machine, Japan, at a speed of 4000 stitches/min and producing a balance seam. 
TABLE 2: Specifications of sewing threads.

\begin{tabular}{lcccc}
\hline Sewing thread linear & \multicolumn{3}{c}{ Specifications of sewing threads } \\
density (Tex) & $\begin{array}{c}\text { Twist direction } \\
\text { (ply/single) }\end{array}$ & Tenacity (CN/Tex) & Breaking elongation (\%) & Initial modulus (CN/Tex) \\
\hline 40 & Z/S & 44.73 & 25.78 & 264.45 \\
60 & $\mathrm{Z} / \mathrm{S}$ & 53.13 & 27.15 & 310.11 \\
80 & $\mathrm{Z} / \mathrm{S}$ & 61.14 & 29.14 & 442.1 \\
\hline
\end{tabular}

TABLE 3: Needle dimensions.

\begin{tabular}{lcccc}
\hline Needle size & \multicolumn{2}{c}{ Shoulder diameter $(\mathrm{mm})$} & Needle eye \\
& & Length $(\mathrm{mm})$ & Width $(\mathrm{mm})$ & 0.30 \\
11 & 0.84 & 0.85 & 0.35 & 0.30 \\
14 & 0.90 & 0.95 & 0.30 \\
16 & 1.00 & 1.04 & 0.36 \\
\hline
\end{tabular}

TABLE 4: L9 Orthogonal design of experiment.

\begin{tabular}{|c|c|c|c|c|}
\hline \multirow{2}{*}{ Factors } & \multirow{2}{*}{ Code } & \multicolumn{3}{|c|}{ Levels } \\
\hline & & 1 & 2 & 3 \\
\hline Blend composition (\%) & A & P/C: 35/65 & $\mathrm{P} / \mathrm{C}: 15 / 85$ & Cotton $100 \%$ \\
\hline Needle thread tension & $\mathrm{B}$ & 75 & 100 & 125 \\
\hline Needle size (Organ make) & $\mathrm{C}$ & 11 & 14 & 16 \\
\hline Sewing thread linear density (Tex) & $\mathrm{D}$ & 40 & 60 & 80 \\
\hline
\end{tabular}

P: polyester; C: cotton.

Any wrinkled fabrics are ironed before preparation of the seam specimen. The seam specimens are prepared in such a way that the existing seams ran through the middle of each specimen. Stitch density is measured on the sewn fabrics by means of a magnifying glass and an accurately marked scale ASTM-D1683 [11]. Similar sewing thread is used as needle thread and the bobbin thread of machine for preparing the seams. During sample preparation the static bobbin thread tension is kept constant. The needle thread tension values are measured by using strain gauge type digital tension meter.

\subsection{Methods}

2.3.1. Experimental Design. The individual and interactive effects of needle size, needle thread tension, and sewing thread linear density on seam quality critical dimensions of suiting fabrics are studied at three levels using L9 orthogonal design. The actual values of different parameters corresponding to coded levels are mentioned in Table 4.

Through the use of L9 orthogonal design, information on single linear effects, single quadratic effects, along with the two factor linear interaction can be obtained. In the present case, 4-factor, L9 orthogonal experimental design at three levels is chosen as it can adequately fulfill the present requirement with only 9 treatment (experiment) combinations wherein parameters can be kept at 3 levels. For effective statistical analysis and validity of inferences, the
TABLE 5: Layout of the experiment in standard order.

\begin{tabular}{lcccc}
\hline Sl. no. & $\begin{array}{c}\text { Fabric blend } \\
\text { composition } \\
\%(\mathrm{~A})\end{array}$ & $\begin{array}{c}\text { Needle } \\
\text { thread } \\
\text { tension (B) }\end{array}$ & $\begin{array}{c}\text { Needle size } \\
(\mathrm{C})\end{array}$ & $\begin{array}{c}\text { Sewing } \\
\text { thread linear } \\
\text { density (D) }\end{array}$ \\
\hline 1 & 1 & 1 & 1 & 1 \\
2 & 1 & 2 & 2 & 2 \\
3 & 1 & 3 & 3 & 3 \\
4 & 2 & 1 & 2 & 3 \\
5 & 2 & 2 & 3 & 1 \\
6 & 2 & 3 & 1 & 2 \\
7 & 3 & 1 & 3 & 2 \\
8 & 3 & 2 & 1 & 3 \\
9 & 3 & 3 & 2 & 1 \\
\hline
\end{tabular}

samples are prepared in a standard order; $1,2,3,4,5,6,7,8$, and 9 as shown in Table 5 .

Conclusively, the testing samples are prepared by changing the parameters, that is, needle thread tension, needle size, and sewing thread linear density, at three levels as per L9 orthogonal design methodology (Table 5) on Juki industrial sewing machine.

The ANOVA technique is conducted to see the effect of individual factors on the seam quality dimensions. The three replicate measures (at different fabric positions from the whole fabric sample lot) are taken, and total degree of freedom is 26 (total combination $9 \times 3=27$; hence, degree of freedom is $27-1=26)$. $F$-ratio and percentage contribution 
TABLE 6: Seam quality characteristics. Response of nine experiments.

\begin{tabular}{|c|c|c|c|c|c|c|c|c|c|c|c|c|c|c|c|c|}
\hline \multirow[t]{2}{*}{$\begin{array}{l}\text { Experiment } \\
\text { number }\end{array}$} & \multicolumn{4}{|c|}{ Factors } & \multicolumn{3}{|c|}{ Seam stiffness $(N)$} & \multicolumn{3}{|c|}{$\begin{array}{l}\text { Seam efficiency (\%) } \\
\text { (Blocks according }\end{array}$} & \multicolumn{3}{|c|}{$\begin{array}{l}\text { Drape coefficient (\%) } \\
\text { o replicate measures) }\end{array}$} & \multicolumn{3}{|c|}{ Seam puckering $(\%)$} \\
\hline & A & $\mathrm{B}$ & $\mathrm{C}$ & $\mathrm{D}$ & 1 & 2 & 3 & 1 & 2 & 3 & 1 & 2 & 3 & 1 & 2 & 3 \\
\hline 1 & 1 & 1 & 1 & 1 & 2.00 & 1.50 & 2.00 & 87 & 86 & 85 & 57 & 55 & 56 & 23 & 21 & 28 \\
\hline 2 & 1 & 2 & 2 & 2 & 1.50 & 1.50 & 2.00 & 81 & 80 & 79 & 53 & 50 & 52 & 48 & 55 & 59 \\
\hline 3 & 1 & 3 & 3 & 3 & 1.50 & 1.50 & 2.00 & 78 & 79 & 77 & 61 & 62 & 60 & 68 & 73 & 70 \\
\hline 4 & 2 & 1 & 2 & 3 & 3.00 & 2.50 & 3.00 & 88 & 86 & 89 & 77 & 74 & 75 & 75 & 77 & 72 \\
\hline 5 & 2 & 2 & 3 & 1 & 3.00 & 3.50 & 3.00 & 63 & 64 & 62 & 70 & 71 & 68 & 37 & 47 & 47 \\
\hline 6 & 2 & 3 & 1 & 2 & 2.50 & 2.50 & 3.50 & 77 & 76 & 75 & 74 & 73 & 72 & 70 & 62 & 57 \\
\hline 7 & 3 & 1 & 3 & 2 & 3.00 & 3.50 & 5.00 & 97 & 97 & 96 & 68 & 66 & 66 & 50 & 50 & 50 \\
\hline 8 & 3 & 2 & 1 & 3 & 3.50 & 3.00 & 3.00 & 79 & 80 & 78 & 68 & 65 & 66 & 69 & 59 & 64 \\
\hline 9 & 3 & 3 & 2 & 1 & 2.50 & 3.00 & 3.50 & 89 & 87 & 90 & 71 & 68 & 68 & 31 & 33 & 36 \\
\hline
\end{tabular}

A: blend composition; B: needle thread tension; C: needle size; D: sewing thread linear density (Tex value).

of different factors are studied. Finally regression equations (Table 8) relating the main factors with seam quality dimensions and response surface regression analysis are established. From the ANOVA analysis, \% contribution of different factors is evaluated based on the following expression:

$$
\text { contribution }(\%)=\frac{\left[\mathrm{SS}_{f}-\left(\mathrm{df}_{f} \times \mathrm{MS}_{e}\right)\right]}{\mathrm{SS}_{t}} \times 100
$$

where $\mathrm{SS}_{f}$ is sum of square of the factor, $\mathrm{df}_{f}$ is degree of freedom of the factor, $\mathrm{MS}_{e}$ is mean square of the error, and $\mathrm{SS}_{t}$ is total sum of squares.

Sewn fabrics are tested for seam stiffness on circular bend stiffness tester as per ASTM standard D 4032-94 [11]. The sample of 8 inch $\times 4$ inch is cut from the fabric and sewn in warp direction with seam allowance of 1 inch; the short side of the specimen must be parallel to the machine (length) direction of the fabric. The air pressure control to the actuator is kept at 60 psi to confirm the tester plunger speed of $1.7 \pm$ $0.3 \mathrm{~s}$ under no load conditions. Ten readings are carried out for each sample.

Sewn fabrics are tested for seam efficiency on Instron tensile testing machine at a speed of $305 \mathrm{~mm} / \mathrm{min}$ and $75 \mathrm{~mm}$ gauge length as per ASTM-D1683. Ten tests are conducted for each sample. For seam strength testing, the sample of 8 inch $\times$ 4 inch is cut from the fabric and sewn in warp direction with seam allowance of 1 inch. Consider

$$
\text { seam efficiency }(\%)=\frac{\text { seam tensile strength }}{\text { fabric tensile strength }} \times 100 \text {. }
$$

Sewn fabrics are tested for drape coefficient on drape meter. Most garments are sewn in the warp direction. Hence, experimental fabric seams are sewn in the warp direction. After seaming, samples are cut into $25 \mathrm{~cm}$ diameter round shapes with the seam positioned across the specimen diameter [12]. The drape coefficient can be calculated as:

$$
\text { drape coefficient }(\%)=\frac{A_{d}-A_{1}}{A_{2}-A_{1}} \times 100
$$

where $A_{d}$ is the area of draped shadow, $A_{1}$ is the area of the disc, and $A_{2}$ is the area of the fabric.

Seam puckering has been calculated by measuring the fabric and seam thickness under constant compressive load of $2 \mathrm{KPa}$ (ISO 9073-2:1997-02). Ten readings are carried out for each sample and puckering calculated by the following formula:

$$
\begin{aligned}
& \text { seam puckering (i.e., thickness strain, \%) } \\
& =\frac{T_{s}-2 T}{2 T} \times 100
\end{aligned}
$$

where $T_{s}$ is the seam thickness in $\mathrm{mm}$ and $T$ is fabric thickness in $\mathrm{mm}$.

\section{Results and Discussion}

3.1. Study of the Seam Stiffness. It is observed from Table 6 that with the replicate measures seam stiffness is changing for all experimental runs. The main factor influencing the seam stiffness is fibre \%, the percentage contribution being 67\% (Table 7). It is also observed from Figure 1 that with the change in blend composition the seam stiffness increases. The increasing trend of seam stiffness with the change in blend percentage is due to the fact that the polyester fibre content goes on decreasing which has low bending stiffness in low load regions (flexural rigidity of polyester fibre is $0.30 \mathrm{mN} \cdot \mathrm{mm}^{2} / \mathrm{tex}^{2}$ ). On the contrary, cotton dominated suiting fabrics has high flexural rigidity of $0.53 \mathrm{mN} \cdot \mathrm{mm}^{2} / \mathrm{tex}^{2}$. So the polyester dominated suiting fabrics show less resistance when bent by the external force encountered during fabric manipulation in spreading and sewing. Hence, fibre types and \% largely influence the seam stiffness (Table 7). However, the effect of sewing thread linear density on seam stiffness is statistically insignificant for medium weight suiting fabrics.

3.2. Study of Seam Strength Efficiency. Figure 2 showed the effect of blend composition and needle thread tension on the seam strength efficiency. As fabric strength increases, the seam strength efficiency decreases because it is inversely 
TABLE 7: ANOVA results. F-value and contribution (\%) of factors on seam characteristics.

\begin{tabular}{|c|c|c|c|}
\hline Characteristics & Factors & $F$-value & $\%$ contribution \\
\hline \multirow{4}{*}{ Seam stiffness $(N)$} & Blend & 35.92 & 67 \\
\hline & Needle thread tension & Not significant & Very small \\
\hline & Needle size & Not significant & Very small \\
\hline & Sewing size & Not significant & Very small \\
\hline \multirow{4}{*}{ Seam efficiency (\%) } & Blend & 308.3 & 32 \\
\hline & Needle thread tension & 510.1 & 53 \\
\hline & Needle size & 85.9 & 9 \\
\hline & Sewing thread linear density & 48.86 & 5 \\
\hline \multirow{4}{*}{ Drape coefficient (\%) } & Blend & 591.6 & 85 \\
\hline & Needle thread tension & 55.6 & 7.8 \\
\hline & Needle size & Not significant & Very small \\
\hline & Sewing thread linear density & 31.9 & 4 \\
\hline \multirow{4}{*}{ Seam puckering (\%) } & Blend & 21.19 & 10 \\
\hline & Needle thread tension & 4.89 & 2 \\
\hline & Needle size & Not significant & Very small \\
\hline & Sewing thread linear density & 157.42 & 80 \\
\hline
\end{tabular}

Not significant at $95 \%$ confidence level.

TABLE 8: Regression equations of seam quality characteristics.

\begin{tabular}{|c|c|c|c|}
\hline Characteristics & Interactive factors & Regression equation & $R^{2}$ \\
\hline Seam stiffness $(N)$ & A-D & $-0.44+2.30 x+0.44 y-0.41 x^{2}+0.08 x y-0.166 y^{2}$ & 0.76 \\
\hline \multirow{2}{*}{ Seam efficiency (\%) } & $A-B$ & $153-33.27 x-50.61 y+9.16 x^{2}-15 x y+11.5 y^{2}$ & 0.98 \\
\hline & A-D & $88-32.44 x+17.33 y+9.16 x^{2}-0.41 x y-3.83 y^{2}$ & 0.97 \\
\hline Drape coefficient $(\%)$ & $A-B$ & $28.41+49.9 x-14.92 y-10.80 x^{2}-0.58 x y+4.22 y^{2}$ & 0.99 \\
\hline Seam puckering (\%) & A-D & $-50.9+54.4 x+41.81 y-11.6 x^{2}-4 x y-3.91 y^{2}$ & 0.96 \\
\hline
\end{tabular}

A: blend composition; B: needle thread tension; C: needle size; D: sewing thread linear density.

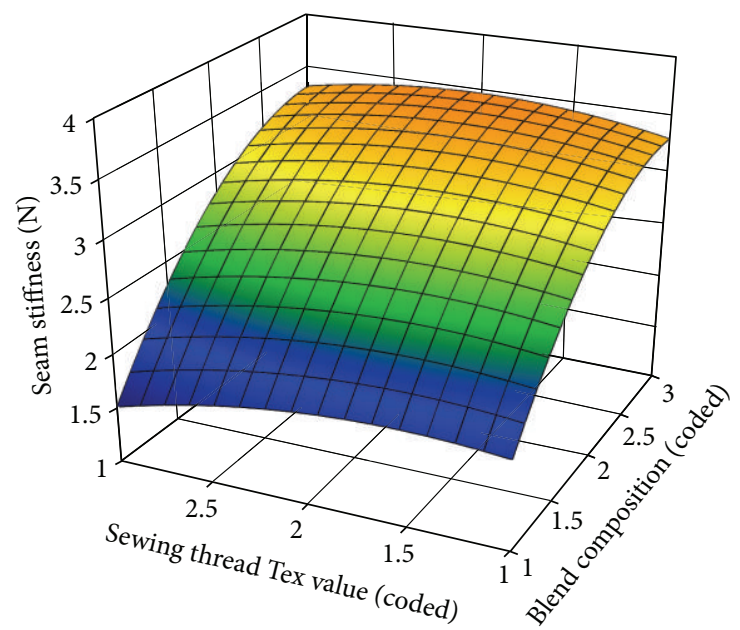

FIGURE 1: Effect of blend composition and sewing thread linear density (Tex value) on seam stiffness.

proportional to the fabric strength for a given sewing thread. The low seam strength efficiency for the polyester dominated suiting fabrics is attributed to the fact that the polyester based suiting fabrics have high extensibility (elongation at break-30\%) and tenacity ( $45 \mathrm{gf} /$ tex) as compared to cotton dominated fabrics (elongation at break-7\%) and tenacity (35 gf/tex). Similarly, in seamed conditions this high strength and elongation is not desirable, hence, results in low seam efficiency for polyester dominated fabrics. The latter decreasing trend for the seam efficiency is due to the less uniform fibre matrix which may lead to fibre slippage and yarn failure. As the fibre matrix becomes uniform in cotton dominated fabrics (100\% cotton), the seam efficiency increases as shown in Figure 2.

The needle thread tension has significant effect on seam efficiency, and the low needle thread tension during stitching improves the seam strength and, hence, seams efficiency considerably; the percentage contribution of needle thread tension is $53 \%$ (Table 7). This is due to some flexibility at the seam to improve the seam strength under sudden stress. However, at high needle thread tension, the fabric has been pulled at the seam leading to puckered, unstable seam resulting in low seam strength efficiency.

It has also been observed that the sewing thread linear density has considerable effect on the seam efficiency; the percentage contribution is 5\% (Table 7). Seam efficiency increases with the sewing thread linear density. This is due to the fact that more number of fibres is incorporated in the coarser sewing thread resulting in high seam strength. 


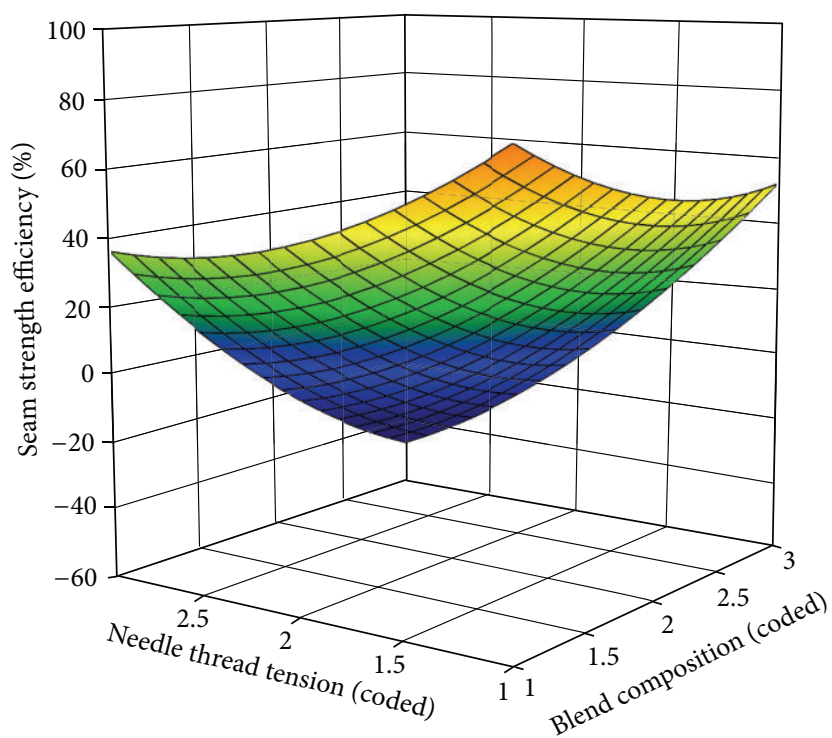

FIGURE 2: Effect of blend composition and needle thread tension on seam strength efficiency.

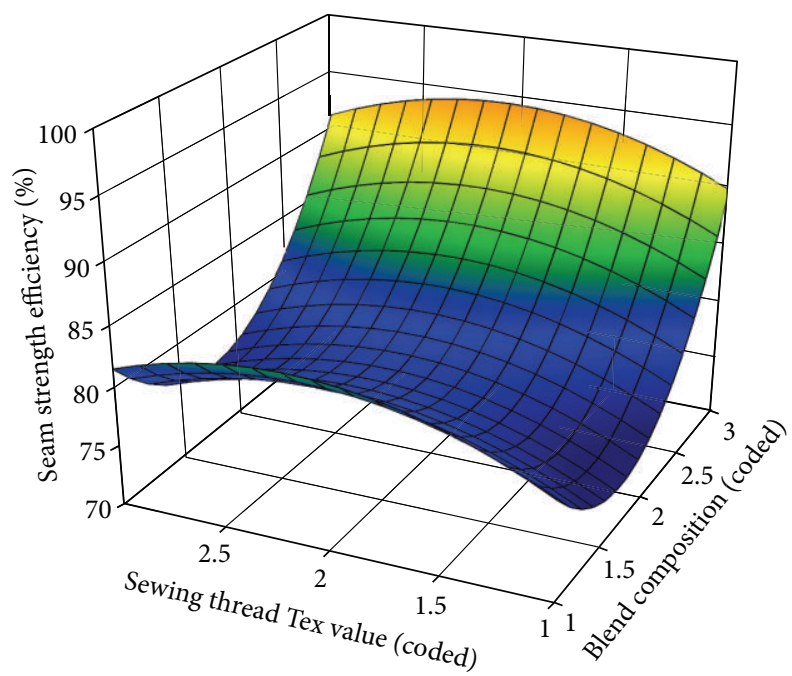

FIGURE 3: Effect of blend composition and sewing thread linear density (Tex value) on seam strength efficiency.

Figure 3 showed also the effect of blend and sewing thread Tex value on seam strength efficiency. The seam efficiency first decreases and then increases considerably with change in blend composition.

The coarser sewing thread has better gripping with the bulkier and resilient polyester fibre component (density $1.40 \mathrm{~g} / \mathrm{cc}$ ) in the polyester suiting fabrics leading to high seam strength efficiency (Figure 3), but the later decreasing trend in seam efficiency is due to the reason of fabric structural jamming in cotton dominated fabrics leading to high fabric strength and low seam efficiency.

3.3. Study of the Draping Behavior. It is observed from Figure 4 that the blend composition of fabric has drastic effect

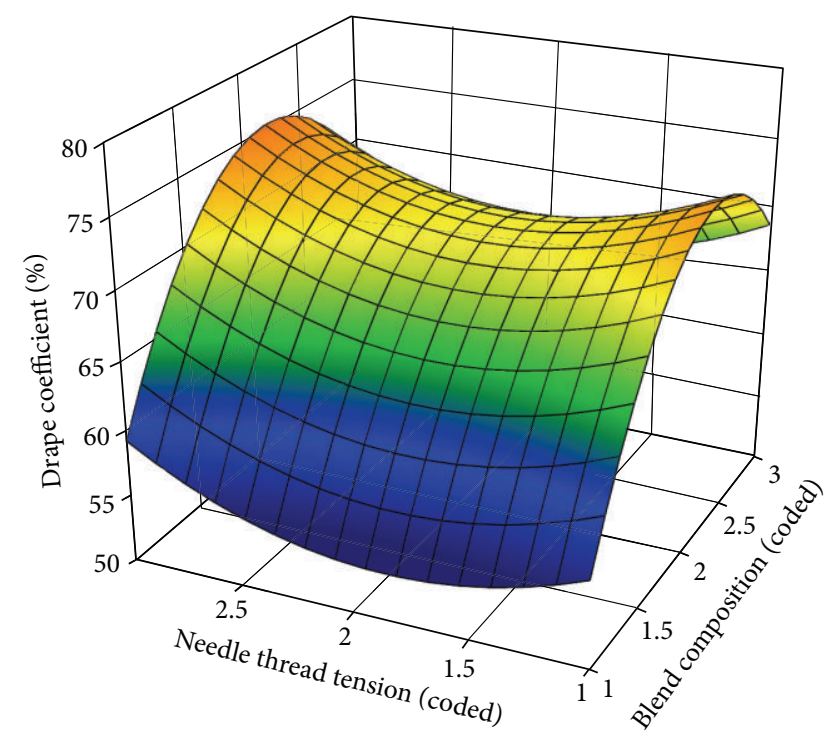

FIGURE 4: Effect of blend composition and needle thread tension on drape coefficient.

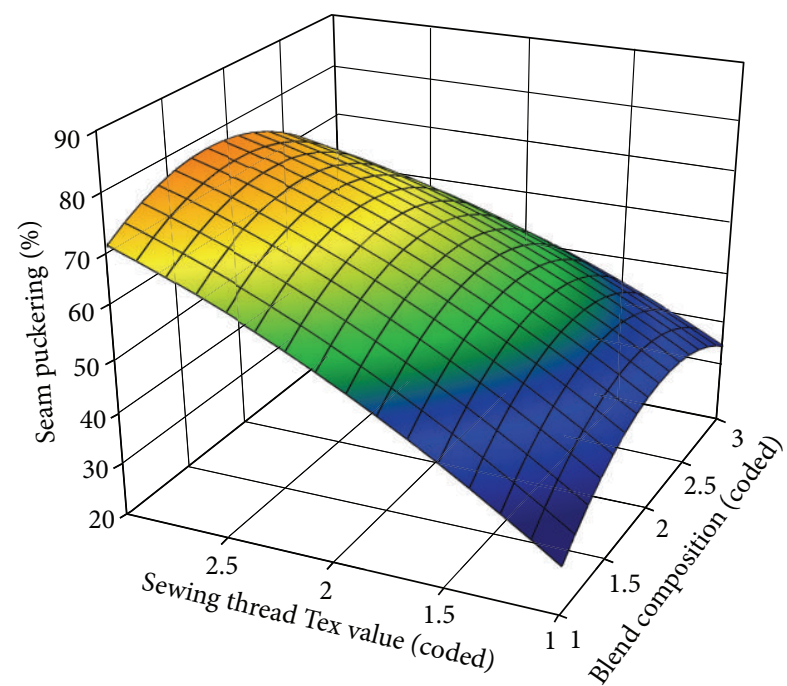

FIGURE 5: Effect of blend composition and sewing thread linear density (Tex value) on seam puckering.

on the drape coefficient (\%); the percentage contribution is $85 \%$ (Table 7). The low-stress extensibility for P/C:65/35 is high which determines the mobility of threads within the fabrics. Higher low-stress extensibility gives more wearing comfort, high drape ability, and consequently low drapecoefficient for polyester dominated suiting fabrics. As the cotton composition goes on increasing the drape coefficient increases in $\mathrm{P} / \mathrm{C}: 15 / 85$ since the bending stiffness of the cotton fibre is higher than the polyester fibre. The later decreasing trend of drape coefficient in cotton dominated suiting fabrics is due to the uniform fibre matrix in $100 \%$ cotton fabrics. However, the other factors needle thread tension and sewing thread linear density have negligible effect on draping behavior. 
3.4. Study of the Seam Puckering. It is observed from Figure 5 that seam puckering is highly dependent on sewing thread linear density, the contribution being $80 \%$ (Table 7). This indicates that the coarser yarn causes the fabric structural jamming and leads to low flexibility at the seam causing seam puckering.

The polyester-based suiting fabrics with the extensible polyester component give higher extensibility in the lowload region; hence, the fabric dimensional stability is reduced which may lead to high seam puckering. The latter decreasing trend in cotton-based suiting fabric is due to the fact that the increase in fabric stiffness leads to the increase in in-plane compression resistance which causes the decrease in seam puckering value.

\section{Conclusions}

(i) The polyester dominated suiting fabrics give minimum seam stiffness because polyester component has low flexural rigidity.

(ii) Seam strength efficiency is high for the uniform fibre matrix in the textile structure.

(iii) At high needle thread tension, seam strength efficiency is low because fabric has been pulled leading to puckered and unstable seam.

(iv) The higher low-stress extensibility of polyester-cotton suiting fabrics $\mathrm{P} / \mathrm{C}: 65 / 35$ is high which gives high drapeability and wearing comfort.

(v) Seam pucker is most effective dimension for evaluating seam quality; bending stiffness has considerable effect on seam puckering.

(vi) Seam puckering and seam strength efficiency increase with the increase in sewing thread linear density

(vii) The cotton dominated suiting fabrics have less seam puckering due to increase in fabric stiffness which leads to increase in in-plane compression resistance.

\section{References}

[1] H. Carr and B. Latham, The Technology of Clothing Manufacturing, Blackwell Scientific, Oxford, UK, 1995.

[2] B. K. Behera and S. Sharma, "Low-stress behaviour and sewability of suiting and shirting fabrics," Indian Journal of Fibre and Textile Research, vol. 23, no. 4, pp. 233-241, 1998.

[3] K. P. S. Cheng and K. P. W. Poon, "Seam properties of woven fabrics," Textile Asia, vol. 33, no. 3, pp. 30-34, 2002.

[4] V. Dobilaite and M. Juciene, "The influence of mechanical properties of sewing threads on seam pucker," International Journal of Clothing Science and Technology, vol. 18, no. 5, pp. 335-345, 2006.

[5] D. H. Brain, "The prediction of strength of lockstitch seams in woven fabric," Journal of Textile Institute, vol. 61, no. 10, pp. 493505, 1970.

[6] N. Tarafder, R. Karmakar, and M. Mondal, "The effect of stitch density on seam performance of garments stitched from plain and twill fabrics," Man-Made Textiles in India, vol. 50, no. 8, pp. 298-302, 2007.
[7] B. Meric and A. Durmaz, "Effect of thread structure and lubrication ratio on seam properties," Indian Journal of Fibre and Textile Research, vol. 30, no. 3, pp. 273-277, 2005.

[8] J. Ukponmwan, A. Mukhopadhyay, and K. N. Chatterjee, "Sewing threads," Textile Progress, vol. 30, no. 3-4, pp. 1-91, 2000.

[9] R. S. Rengasamy, V. K. Kothari, R. Alagirusamy, and S. Modi, "Studies on air-jet textured sewing threads," Indian Journal of Fibre and Textile Research, vol. 28, no. 3, pp. 281-287, 2003.

[10] G. Sundaresan, K. R. Salhotra, and P. K. Hari, "Strength reduction in sewing threads during high speed sewing in industrial lockstitch machine. Part II: effect of thread and fabric properties," International Journal of Clothing Science and Technology, vol. 10, no. 1, pp. 64-79, 1998.

[11] http://www.astm.org/.

[12] J. E. Booth, Principles of Textile Testing, CBS, 1996. 

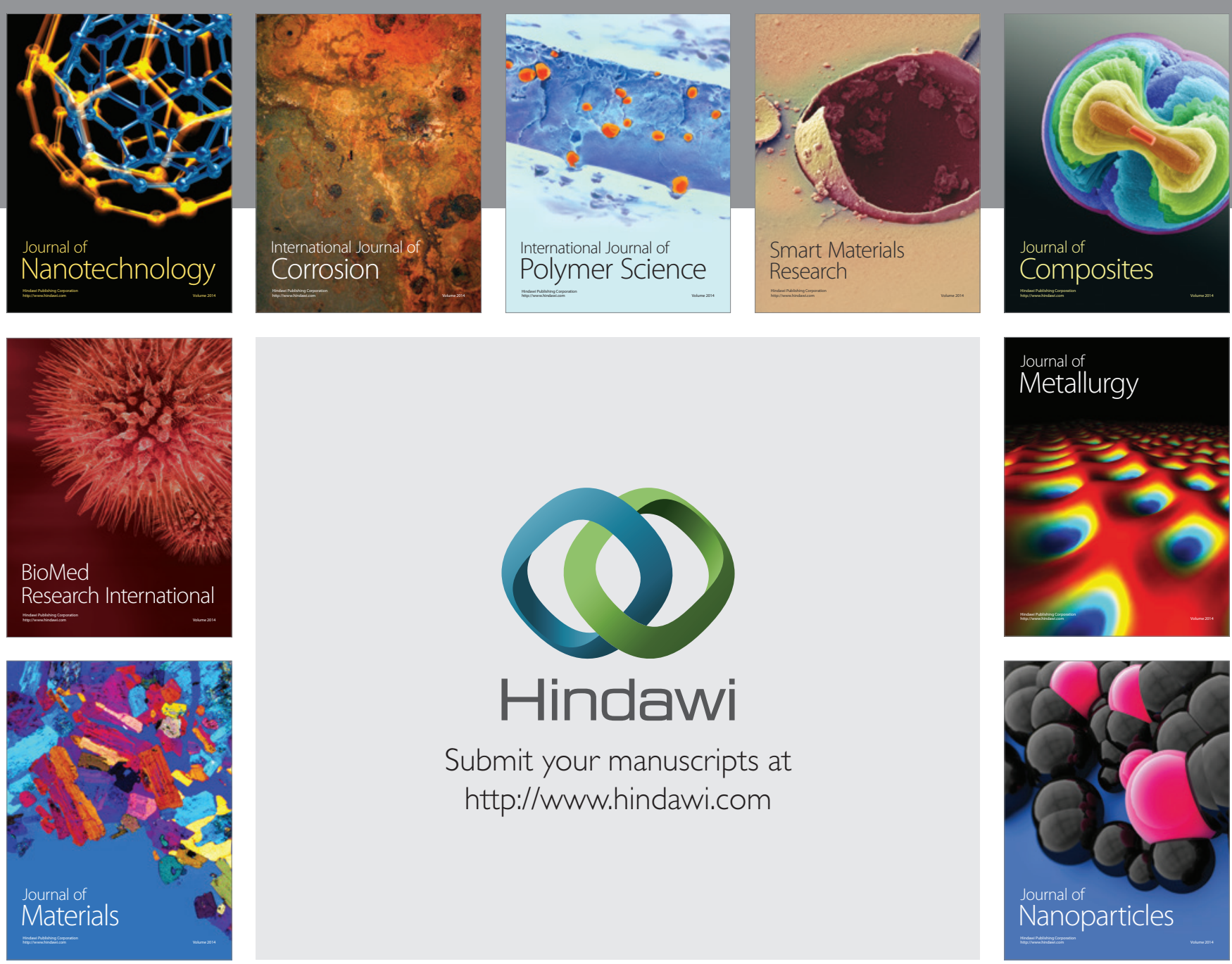

Submit your manuscripts at http://www.hindawi.com
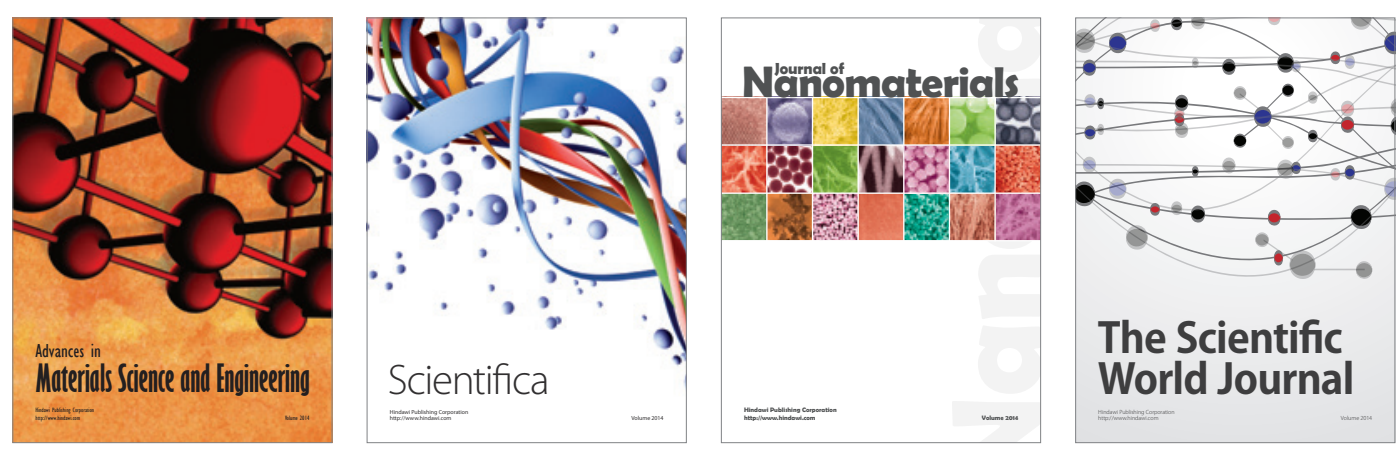

\section{The Scientific World Journal}
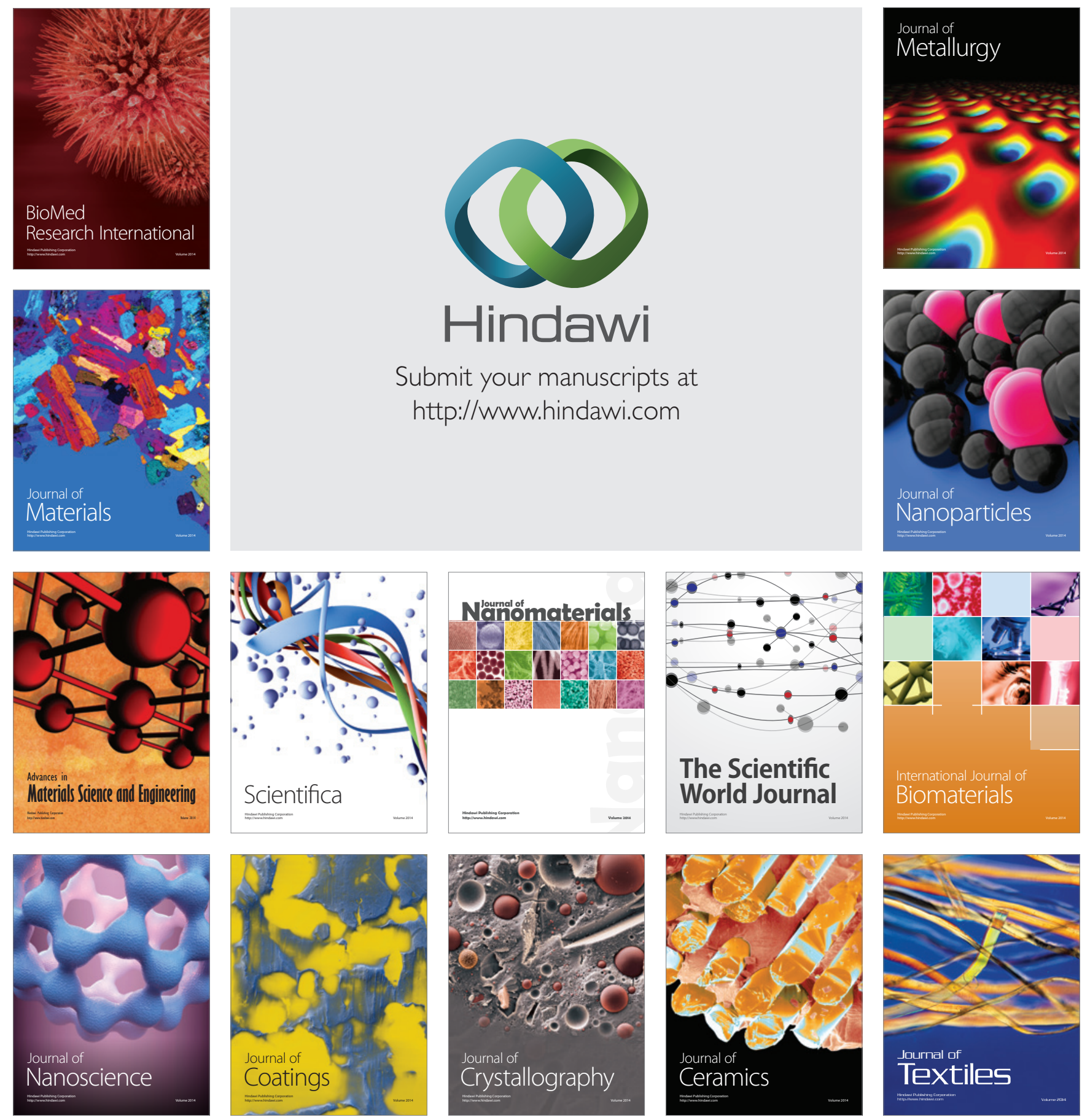